\title{
Clinical Utility of Eribulin Mesylate in the Treatment of Breast Cancer: A Chinese Perspective
}

This article was published in the following Dove Press journal: Breast Cancer: Targets and Therapy

\section{Peng Yuan \\ Binghe $X u(D)$}

National Cancer Center, National Clinical Research Center for Cancer, Cancer Hospital, Chinese Academy of Medical Sciences and Peking Union Medical College, Beijing, I0002I, People's Republic of China
Correspondence: Binghe $\mathrm{Xu}$

National Cancer Center, National Clinical Research Center for Cancer, Cancer Hospital, Chinese Academy of Medical Sciences and Peking Union Medical College, Beijing, I0002I, People's

Republic of China

Tel +86 1087788826

Email xubinghebm@।63.com

\begin{abstract}
Eribulin mesylate, a synthetic derivative of the anti-mitotic agent halichondrin B, has a unique tubulin-based mechanism of action that is distinct from other anti-microtubule agents including taxanes and vinca alkaloids. Consistent with this unique activity, eribulin has shown clinical efficacy in patients with metastatic breast cancer (MBC) that progressed following prior taxane and anthracycline therapy. The evidence presented in this review indicates that eribulin represents a treatment option for patients with HER2-negative metastatic breast cancer. Improved survival outcomes and better tolerability compared with vinorelbine supported the first approval of eribulin in China in 2019; eribulin was approved for women with locally advanced/metastatic HER2-negative breast cancer after treatment failure with at least two chemotherapy regimens, including an anthracycline and a taxane. Eribulin has also shown promising efficacy in patients with HER2-positive advanced breast cancer when used in combination with trastuzumab or pertuzumab, and subgroup analyses from the Phase III clinical trials support the continued evaluation of eribulin in patients with triple-negative disease. The unique non-mitotic effects of eribulin, including vascular remodeling, coupled with its clinical efficacy and safety profile, may permit the broader use of this agent in patients with MBC.
\end{abstract}

Keywords: eribulin mesylate, breast cancer, China

\section{Introduction}

Breast cancer is the most common cancer diagnosis in women in China, ahead of lung, bronchus and gastrointestinal cancers. ${ }^{1}$ Longitudinal age-incidence studies have indicated similar incidence rates in Western and Asian populations; however, breast cancer incidence rates among younger generations in Asian countries may be surpassing rates in the United States. ${ }^{2}$ The most recent breast cancer statistics for China indicate an estimated 268,600 new cases and 69,500 deaths in 2015. ${ }^{1}$

Breast cancer is also a leading cause of cancer death (after lung and gastrointestinal cancers) in Chinese women overall, and the leading cause of cancer death in Chinese women younger than 45 years of age. ${ }^{1}$ A study of trends in mortality from Central China reported an age-standardized mortality rate for breast cancer of 7.5 per 100,000 persons in 2014. ${ }^{3}$ A significant upward trend in mortality was also found in patients aged 55-79 years between 1990 and 2014 and this was projected to continue through to 2024 .

Patients diagnosed with metastatic breast cancer (MBC) have a poor prognosis. Data from the US reveal the 5-year relative survival rate for $\mathrm{MBC}$ is around $28 \%$, 
compared with $99 \%$ in patients diagnosed with localized disease. ${ }^{4}$ Treatment options for $\mathrm{MBC}$, including chemotherapy, endocrine therapy and biologic therapy are largely palliative with the aim of prolonging survival and maintaining quality of life. ${ }^{5}$ Chemotherapy is widely used in patients with metastatic disease; however, drug resistance is a common problem in later disease stages, often requiring alternative treatment options. Therefore, identifying novel treatments for patients with resistant disease and difficult-to-treat tumor types such as human epidermal growth factor receptor 2 (HER2)-negative and triplenegative $(\mathrm{TN})$ breast cancer are urgently needed to address the high mortality rate.

Eribulin mesylate is an anti-mitotic agent with a unique tubulin-based mechanism of action that has shown significant clinical activity in patients with MBC. It was approved in China in 2019 for treatment of patients with locally recurrent or metastatic disease who have received prior anthracycline and paclitaxel therapy.

This review provides an overview of the unique mechanism of action of eribulin which may, at least in part, explain the activity seen with this agent in patients with disease that has progressed on prior anthracycline and taxane therapy. We also summarize efficacy and safety data from clinical trials, supporting the use of eribulin in MBC, including pivotal Phase III data from China. Eribulin has also been assessed in the (neo)adjuvant setting and these clinical data are also briefly included for completeness. Finally, we discuss current treatment recommendations for MBC in China, and consider potential future uses of eribulin in the clinic.

\section{Search Methodology}

A literature search was performed using www.Pubmed.gov for literature relevant to the use of eribulin in the treatment of metastatic breast cancer (eribulin AND "metastatic breast cancer"). All studies appearing in Pubmed.gov prior to completion of the first draft (30 June, 2020) were considered for inclusion. The resulting literature was evaluated for inclusion based on relevance to the treatment of metastatic breast cancer with a focus on use in China. Separate literature searches were conducted to provide an overview of the mechanism of action of eribulin and to identify real-world evidence regarding the use of eribulin in this setting.

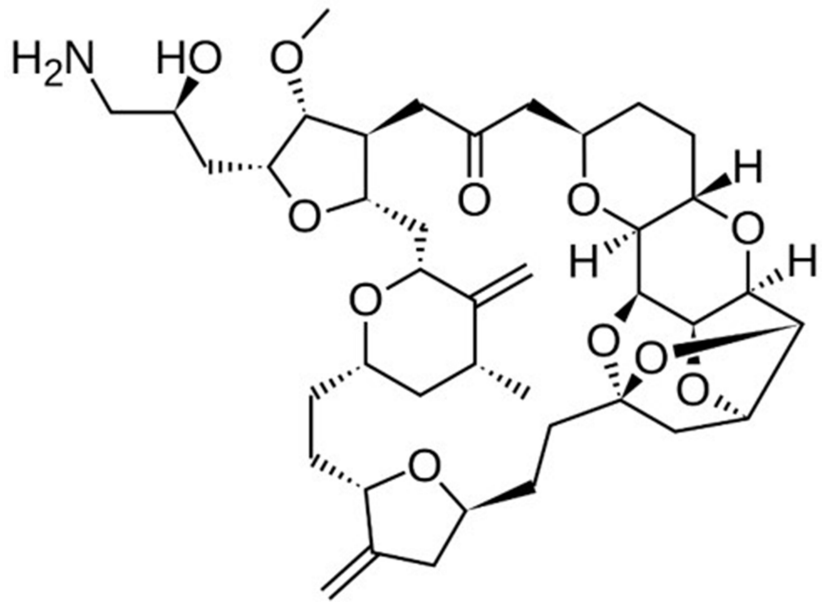

Figure I Chemical structure of eribulin. Reproduced from Wikipedia. Avalable from: https://en.wikipedia.org/wiki/Eribulin. Creative Commons AttributionShareAlike 3.0 Unported License: https://creativecommons.org/licenses/by-sa/3.0/. ${ }^{62}$

\section{Eribulin Mechanism of Action}

Eribulin mesylate, a synthetic derivative of halichondrin B (Figure 1), has demonstrated significant antitumor activity across a broad range of human cancer cell lines in vitro, including in paclitaxel-resistant cell lines. ${ }^{6-8}$ Marked tumor regression has also been shown to occur with eribulin in xenograft models of various human cancers, including breast cancer. ${ }^{6,7}$

Eribulin's potent and broad antitumor activity appears to be attributed to both anti-mitotic and non-mitotic mechanisms of action. Like other microtubule-targeting agents such as taxanes and vinca alkaloids, eribulin disrupts microtubule dynamics, that play a key role in many cellular processes including cell proliferation. ${ }^{9}$ However, the binding site and consequent mechanism of action of eribulin are distinct from these other agents and the antimitotic effects of eribulin are irreversible. ${ }^{8,10-14}$ This unique mode of action may explain the antitumor activity observed in vitro with eribulin in taxane-resistant tumor cells harboring $\beta$-tubulin mutations. ${ }^{8,12,15}$

Non-mitotic effects of eribulin include vascular remodeling (increased vascular perfusion, reduced hypoxia) and reversal of the epithelial-to-mesenchymal (EMT) phenotype, which is known to promote local tumor invasiveness and metastasis. ${ }^{16}$ In general, the unique eribulin binding sites suggest that efficacy may be retained in patients with previous exposure to other taxane-based treatments and the non-mitotic effects of eribulin permit its use in combination with other therapies. 
Table I Summary of Eribulin Phase III Clinical Trials in Locally Advanced/Metastatic Breast Cancer

\begin{tabular}{|c|c|c|c|}
\hline & $\begin{array}{l}\text { Median OS, Months ( } 95 \% \\
\text { CI) }\end{array}$ & $\begin{array}{l}\text { Median PFS, Months }(95 \% \\
\mathrm{Cl})\end{array}$ & $\begin{array}{l}\text { Objective Response } \\
\text { Rate, \% }\end{array}$ \\
\hline \multicolumn{4}{|l|}{ Cortes et al $20 \mathrm{II}^{17}$} \\
\hline Eribulin $\left(1.4 \mathrm{mg} / \mathrm{m}^{2} \mathrm{dl}, 8 \mathrm{q} 2 \mathrm{Id} ; \mathrm{n}=508\right)^{\mathrm{a}}$ & |3.1 (|I.8-14.3) & $3.7(3.3-3.9)$ & $12.2(9.4-15.5)$ \\
\hline \multirow[t]{2}{*}{ TPC $(n=254)^{a}$} & $10.6(9.3-12.5)$ & $2.2(2.1-3.4)$ & $4.7(2.3-8.4)$ \\
\hline & $P=0.04^{b}$ & $P=0.137^{b}$ & $P=0.002^{c}$ \\
\hline \multicolumn{4}{|l|}{ Kaufmann et al $2015^{18}$} \\
\hline Eribulin $\left(1.4 \mathrm{mg} / \mathrm{m}^{2} \mathrm{dl}, 8 \mathrm{q} 2 \mathrm{Id} ; \mathrm{n}=554\right)^{\mathrm{c}}$ & $15.9(15.2-17.6)$ & $4.1(3.5-4.3)$ & $11.0(8.5-13.9)$ \\
\hline \multirow{2}{*}{$\begin{array}{l}\text { Capecitabine }\left(1.25 \mathrm{mg} / \mathrm{m}^{2} \text { bid dl-14 q2 Id; }\right. \\
\mathrm{n}=548)^{\mathrm{c}}\end{array}$} & $14.5(13.1-16.0)$ & $4.2(3.9-4.8)$ & $11.5(8.9-14.5)$ \\
\hline & $P=0.056^{b}$ & $\mathrm{P}=0.30^{\mathrm{b}}$ & $\mathrm{P}=0.85^{\mathrm{c}}$ \\
\hline \multicolumn{4}{|l|}{ Yuan et al $2019^{19}$} \\
\hline Eribulin (I.4 mg/m² dI,8 q2I d; n=264) & $2.8(2.8-4.1)$ & |3.4 (| | . $5-16.2)$ & $30.7(25.2-36.6)$ \\
\hline \multirow[t]{2}{*}{ Vinorelbine $\left(25 \mathrm{mg} / \mathrm{m}^{2} \mathrm{dl}, 8,15 \mathrm{q} 2 \mathrm{Id} ; \mathrm{n}=266\right)$} & $2.8(2.7-2.8)$ & $12.5(10.6-16.6)$ & $16.9(12.6-22.0)$ \\
\hline & $\begin{array}{l}\mathrm{HR}, 0.80 ; 95 \% \mathrm{Cl}: 0.65-0.98 \\
P=0.036^{\text {b, d }}\end{array}$ & $P=0.838^{b}$ & $\mathrm{P}<0.00 \mathrm{I}^{\mathrm{e}}$ \\
\hline
\end{tabular}

Notes: ${ }^{a}$ Patients had recurrent or $M B C$ and had received $\geq 2$ prior chemotherapy regimens for advanced disease, including an anthracycline and a taxane ( $n=468$ patients were eribulin RE, and 214 were TPC RE). ${ }^{b}$ Log rank test. ${ }^{\mathrm{C}}$ Fisher's exact test. ${ }^{\mathrm{d}}$ Hazard ratio $0.8,95 \% \mathrm{Cl}, 0.65-0.98, \mathrm{P}=0.036$. ${ }^{\mathrm{e}} \mathrm{Cochran}-\mathrm{mantel}$-haenszel. Patients had locally advanced or $\mathrm{MBC}$ and had received up to 2 prior chemotherapy regimens for metastatic disease, including an anthracycline and a taxane (68\% were HER2-negative and approximately $25 \%$ had TN disease).

Abbreviations: Bid, twice daily; $\mathrm{Cl}$, confidence interval; d, day; HR, hazard ratio; MBC, metastatic breast cancer; mo, months; OS, overall survival; PFS, progression-free survival; q2Id, every 3 weeks; RE, response evaluable; TN, triple negative; TPC, treatment of physician's choice.

\section{Clinical Studies of Eribulin in Women with Locally Recurrent/ Metastatic Breast Cancer}

The clinical efficacy and tolerability of eribulin in women with locally recurrent/metastatic breast cancer has been studied in three large, randomized Phase III trials; two conducted predominantly in the US, Europe and Australia (international studies), ${ }^{17,18}$ and one study conducted in China. ${ }^{19,20}$ Patients in these studies had locally advanced or metastatic disease and typically had received at least two prior chemotherapy regimens including an anthracycline and a taxane. In the international studies, eribulin was compared with treatment of physician's choice (TPC) or capecitabine and in the Chinese study vinorelbine was the comparator. The results of these trials are summarized in Table 1 and discussed below. The findings of subgroup analyses in patients with HER2positive, HER2-negative, estrogen receptor (ER)-negative and TN disease are also described, and summarized in Table 2.

\section{International Phase III Studies of Eribulin in Locally Advanced/Metastatic Breast Cancer}

Eribulin significantly improved overall survival (OS) versus TPC in heavily pretreated women enrolled in the EMBRACE trial (NCT00388726), ${ }^{17}$ although it did not provide a significant improvement in OS versus capecitabine in study E7389-G000-301 (NCT00337103; Table 1). ${ }^{18}$ Subgroup analyses in the latter trial indicated longer OS with eribulin versus capecitabine in patients with HER2-negative, ER-negative, or TN disease (Table 2). ${ }^{21}$

Pooled analyses of these two Phase III studies reported significantly improved survival outcomes with eribulin versus comparators in heavily pretreated patients ${ }^{22-24}$ and also support subgroup analyses from the E7389-G000-301 study that indicate survival benefit in specific patient subgroups (although no OS benefit was observed in the overall study population). ${ }^{21,22,25}$ In particular, median OS was significantly longer with eribulin versus comparator in patients with either HER2-negative or TN disease (Table 2). In contrast, OS was 
Table 2 Summary of Data from Phase II Phase III Studies of Eribulin in Subgroups of Patients with MBC (Including Pooled Analyses of Multiple Trials)

\begin{tabular}{|c|c|c|c|}
\hline & Patient Group & $\begin{array}{l}\text { Treatment Regimen and } \\
\text { Clinical Outcomes }\end{array}$ & Comments \\
\hline \multicolumn{4}{|c|}{$\begin{array}{l}\text { Eribulin plus trastuzumab in HER2-positive MBC } \\
\text { First-line therapy }\end{array}$} \\
\hline $\begin{array}{l}\text { Wilks et al } \\
2014^{30}\end{array}$ & $\begin{array}{l}\text { Locally recurrent/metastatic disease } \\
(n=52)\end{array}$ & $\begin{array}{l}\text { Eribulin + trastuzumab } \\
\text { ORR: } 71 \% \\
\text { DOR: } 11.1 \text { months } \\
\text { PFS: } 11.6 \text { months }\end{array}$ & $\begin{array}{l}\text { Clinical efficacy was not affected by prior adjuvant/ } \\
\text { neoadjuvant trastuzumab therapy }{ }^{32} \\
\text { Efficacy was lower in patients who had received prior } \\
\text { anthracycline/taxane therapy }{ }^{31}\end{array}$ \\
\hline $\begin{array}{l}\text { Sakaguchi } \\
\text { et al } 2018^{33}\end{array}$ & $\begin{array}{l}\text { Locally recurrent/metastatic disease } \\
(\mathrm{n}=28)\end{array}$ & $\begin{array}{l}\text { Eribulin+ trastuzumab } \\
\text { ORR: } 53.6 \% \\
\text { PFS: } 344 \text { days }\end{array}$ & \\
\hline $\begin{array}{l}\text { Inoue et al } \\
2019^{34}\end{array}$ & Metastatic disease $(n=25)$ & $\begin{array}{l}\text { Eribulin + trastuzumab + } \\
\text { pertuzumab } \\
\text { ORR: } 80.0 \% \\
\text { PFS: } 23.1 \text { months }\end{array}$ & \\
\hline $\begin{array}{l}\text { Yamashita } \\
\text { et al. } 2020^{35}\end{array}$ & Advanced or metastatic disease $(n=49)$ & $\begin{array}{l}\text { Eribulin }+ \text { trastuzumab }+ \\
\text { pertuzumab } \\
\text { CR: } 17.4 \% \\
\text { PR: } 43.5 \% \\
\text { PFS: } 9.2 \text { months }\end{array}$ & $\begin{array}{l}\text { Grade } 3 / 4 \text { AEs } \\
\text { Neutropenia: } 10.2 \% \\
\text { Febrile neutropenia: } 4.1 \% \\
\text { Hypertension: } 6.1 \%\end{array}$ \\
\hline \multicolumn{4}{|c|}{$\begin{array}{l}\text { Eribulin in HER2-negative MBC } \\
\text { First-line therapy }\end{array}$} \\
\hline $\begin{array}{l}\text { Mclntyre } \\
\text { et al } \\
2014^{31,39}\end{array}$ & $\begin{array}{l}\text { Locally recurrent/metastatic disease and } \\
\geq 12 \text { months since neo/adjuvant } \\
\text { chemotherapy }(n=56)\end{array}$ & $\begin{array}{l}\text { Eribulin } \\
\text { ORR: } 28.6 \% \text { (all partial } \\
\text { response) } \\
\text { PFS: } 6.8 \text { months }\end{array}$ & $\begin{array}{l}\text { Prior anthracycline and/or taxane therapy did not } \\
\text { affect the response rate }\end{array}$ \\
\hline $\begin{array}{l}\text { Ortega } \\
\text { et al } 2019^{40}\end{array}$ & $\begin{array}{l}\text { Metastatic disease with prior taxane in } \\
\text { earlier disease stage }(n=53)\end{array}$ & $\begin{array}{l}\text { Eribulin } \\
\text { ORR: } 20.8 \% \\
\text { TTP: } 4.1 \text { months }\end{array}$ & $\begin{array}{l}\text { Eribulin had clinical activity in some patients who had } \\
\text { received prior aggressive taxane therapy }\end{array}$ \\
\hline $\begin{array}{l}\text { Takashima } \\
\text { et al } 2016^{41}\end{array}$ & $\begin{array}{l}\text { Locally advanced/metastatic disease } \\
(\mathrm{n}=35)\end{array}$ & $\begin{array}{l}\text { Eribulin } \\
\text { ORR: } 54.3 \% \\
\text { PFS: } 5.8 \text { months } \\
\text { OS: } 35.9 \text { months }\end{array}$ & \\
\hline $\begin{array}{l}\text { Kimura } \\
\text { et al } 2018^{46}\end{array}$ & $\begin{array}{l}\text { First- or second-line locally advanced/ } \\
\text { metastatic disease }(n=35)\end{array}$ & $\begin{array}{l}\text { Eribulin } \\
\text { ORR: } 37.1 \% \\
\text { PFS: } 6.2 \text { months } \\
\text { OS: } 21.4 \text { months }\end{array}$ & \\
\hline $\begin{array}{l}\text { Park et al } \\
2017^{43}\end{array}$ & Metastatic disease $(n=\mid 18)$ & $\begin{array}{l}\text { Eribulin/gemcitabine vs } \\
\text { paclitaxel/gemcitabine } \\
\text { 6-mo PFS: } 72 \% \text { vs } 73 \%(P=N S) \\
\text { OS: NR vs } 21.2 \text { months } \\
\text { (P=NS) } \\
\text { CBR: } 44 \% \text { vs } 49 \%(P=N S)\end{array}$ & $\begin{array}{l}\text { Neurotoxicity was less common with the eribulin } \\
\text { regimen (grade } \geq 2 \text { neurotoxicity } 13.6 \% \text { versus } 45.8 \% \text {; } \\
\mathrm{P}<0.00 \text { I) }\end{array}$ \\
\hline
\end{tabular}

(Continued) 
Table 2 (Continued).

\begin{tabular}{|c|c|c|c|}
\hline & Patient Group & $\begin{array}{l}\text { Treatment Regimen and } \\
\text { Clinical Outcomes }\end{array}$ & Comments \\
\hline $\begin{array}{l}\text { Tolaney } \\
\text { et } \mathrm{al}^{44}\end{array}$ & $\begin{array}{l}\text { Metastatic disease with } 0-2 \text { lines of prior } \\
\text { chemotherapy }(n=88)\end{array}$ & $\begin{array}{l}\text { Eribulin + pembrolizumab } \\
\text { vs pembrolizumab } \\
\text { PFS: } 4.1 \text { vs } 4.2 \text { months; } \mathrm{HR} \text {, } \\
0.80 ; 95 \% \mathrm{Cl}, 0.50-1.26 ; \\
\mathrm{P}=0.33 \\
\text { ORR: } 27 \% \text { vs } 34 \% ; \mathrm{P}=0.49\end{array}$ & $\begin{array}{l}\text { Grade } \geq 3 \text { AEs reported in } 65 \% \text { of all patients } \\
\text { Two treatment-related deaths in the combination } \\
\text { group (attributed to both drugs), due to immune- } \\
\text { related colitis in the setting of sepsis }\end{array}$ \\
\hline \multicolumn{4}{|c|}{ Later therapy lines } \\
\hline $\begin{array}{l}\text { Smith et al } \\
2020^{61}\end{array}$ & $\begin{array}{l}\text { Metastatic disease }(2-5 \text { prior } \\
\text { chemotherapy regimens; } n=58)\end{array}$ & $\begin{array}{l}\text { Eribulin (new biweekly } \\
\text { schedule: days I and I5 of } \\
\text { each } 28 \text {-day cycle) } \\
\text { ORR: } 12 \% \\
\text { PFS: } 3.6 \text { months }\end{array}$ & $\begin{array}{l}\text { Bi-weekly eribulin administration schedule showed } \\
\text { similar safety and efficacy to the standard schedule } \\
\text { (day I and } 8 \text { every } 21 \text { days) }\end{array}$ \\
\hline $\begin{array}{l}\text { Pivot et al } \\
2018^{25}\end{array}$ & $\begin{array}{l}\text { Subgroup analysis (metastatic } \\
\text { disease second-line treatment setting; } \\
n=392 \text { ) }\end{array}$ & $\begin{array}{l}\text { Eribulin vs capecitabine } \\
\text { OS: } 16.1 \text { vs } 13.5 \text { months } \\
(P=0.026)\end{array}$ & $\begin{array}{l}\text { OS was significantly longer with eribulin versus } \\
\text { capecitabine }\end{array}$ \\
\hline $\begin{array}{l}\text { Twelves } \\
\text { et al } 2014^{22}\end{array}$ & $\begin{array}{l}\text { Pooled analysis (heavily pretreated locally } \\
\text { recurrent/metastatic disease; } n=1320 \text { ) }\end{array}$ & $\begin{array}{l}\text { Eribulin vs comparator } \\
\text { OS: } 15.2 \text { vs } 12.3 \text { months } \\
(P=0.002)\end{array}$ & $\begin{array}{l}\text { OS was significantly longer with eribulin versus } \\
\text { comparator } \\
\text { OS was similar between treatment groups in patients } \\
\text { with HER2-negative/HR+ disease }\end{array}$ \\
\hline $\begin{array}{l}\text { Twelves } \\
\text { et al } 2016^{21}\end{array}$ & $\begin{array}{l}\text { Subgroup analysis (heavily pretreated } \\
\text { locally recurrent or metastatic disease; } \\
n=755 \text { ) }\end{array}$ & $\begin{array}{l}\text { Eribulin vs capecitabine } \\
\text { OS: } 15.9 \text { vs } 13.5 \text { months } \\
(P=0.003)\end{array}$ & $\begin{array}{l}\text { OS was significantly longer with eribulin versus } \\
\text { capecitabine }\end{array}$ \\
\hline $\begin{array}{l}\text { Yuan et al } \\
2019^{19}\end{array}$ & $\begin{array}{l}\text { Subgroup analysis in heavily pretreated } \\
\text { patients with locally recurrent/metastatic } \\
\text { disease in China; } n=399 \text { ) }\end{array}$ & $\begin{array}{l}\text { Eribulin vs vinorelbine } \\
\text { PFS: } \mathrm{HR}, 0.76 ; 95 \% \mathrm{Cl} \text {, } \\
0.60-0.96\end{array}$ & PFS was favorable with eribulin versus vinorelbine \\
\hline $\begin{array}{l}\text { Inoue et al } \\
2020^{45}\end{array}$ & $\begin{array}{l}\text { Real world study in Japan in first- } \\
\text {, second-, third- or later line therapy } \\
(n=637)\end{array}$ & $\begin{array}{l}\text { Eribulin } \\
\text { OS: } 15.6 \text { months } \\
\text { TTF } 4.2 \text { months }\end{array}$ & \\
\hline \multicolumn{4}{|c|}{ Eribulin in triple-negative disease } \\
\hline $\begin{array}{l}\text { Twelves } \\
\text { et al } 2014^{22}\end{array}$ & $\begin{array}{l}\text { Pooled analysis in heavily pretreated } \\
\text { locally recurrent/metastatic disease } \\
(n=428)\end{array}$ & $\begin{array}{l}\text { Eribulin vs comparator } \\
\text { OS: } 12.9 \text { vs } 8.2(P=0.006)\end{array}$ & $\begin{array}{l}\text { OS was significantly longer with eribulin versus } \\
\text { comparator }\end{array}$ \\
\hline $\begin{array}{l}\text { Twelves } \\
\text { et al } 2016^{21}\end{array}$ & $\begin{array}{l}\text { Subgroup analysis (heavily pretreated } \\
\text { locally recurrent/metastatic disease; } \\
(n=284)\end{array}$ & $\begin{array}{l}\text { Eribulin vs capecitabine } \\
\text { OS: } 14.4 \text { vs } 9.4 \text { months } \\
(P=0.006)\end{array}$ & $\begin{array}{l}\text { OS was significantly longer with eribulin versus } \\
\text { capecitabine }\end{array}$ \\
\hline $\begin{array}{l}\text { Yuan et al } \\
2019^{19}\end{array}$ & $\begin{array}{l}\text { Subgroup analysis in heavily pretreated } \\
\text { patients with locally recurrent/metastatic } \\
\text { disease in China }(n=\mid 32)\end{array}$ & $\begin{array}{l}\text { Eribulin vs vinorelbine } \\
\text { PFS: } \mathrm{HR}, 0.70 ; 95 \% \mathrm{Cl} \text {, } \\
0.47-1.03\end{array}$ & PFS was favorable with eribulin versus vinorelbine \\
\hline
\end{tabular}

Abbreviations: $A E$, adverse event; $C B R$, clinical benefit rate; $\mathrm{Cl}$, confidence interval; $C R$, complete response; $D O R$, duration of response; HER2, human epidermal growth factor receptor 2; HR, hazard ratio; HR+, hormone receptor positive; MBC, metastatic breast cancer; NR, not reached; NS, not significant; ORR, overall response rate; OS, overall survival; PFS, progression-free survival; PR, partial response; TTF, time to treatment failure; TTP, time to progression. 
Table 3 Treatment Emergent Adverse Events of Any Grade Occurring in $>10 \%$ of Patients or Grade $3 / 4$ Occurring in $>2 \%$ of Patients in Phase III Clinical Trials (Pooled Data from Two Phase III Clinical Trials ${ }^{21,22}$ ) as Presented by Pivot et al $^{27}$

\begin{tabular}{|c|c|c|c|c|c|c|}
\hline & \multicolumn{3}{|c|}{ Eribulin $(n=932)$} & \multicolumn{3}{|c|}{ Comparator $(n=689)$} \\
\hline & Any grade & Grade 3 & Grade 4 & Any Grade & Grade 3 & Grade 4 \\
\hline Any TEAE & 901 (96.7) & $361(38.7)$ & $258(27.7)$ & $629(91.3)$ & $248(36.0)$ & $76(11.0)$ \\
\hline Any serious TEAE & $199(21.4)$ & $83(8.9)$ & $49(5.3)$ & $155(22.5)$ & $60(8.7)$ & $38(5.5)$ \\
\hline Any TEAE leading to discontinuation & $105(11.3)$ & $49(5.3)$ & $10(1.1)$ & $94(13.6)$ & $43(6.2)$ & $17(2.5)$ \\
\hline Neutropenia & $500(53.6)$ & $218(23.4)$ & $208(22.3)$ & $142(20.6)$ & $53(7.7)$ & $20(2.9)$ \\
\hline Alopecia & $361(38.7)$ & $0(0)$ & $0(0)$ & $42(6.1)$ & $0(0)$ & $0(0)$ \\
\hline Nausea & $268(28.8)$ & $7(0.8)$ & $0(0)$ & $176(25.5)$ & $13(1.9)$ & $0(0)$ \\
\hline Peripheral neuropathy ${ }^{a}$ & $266(28.5)$ & $64(6.9)$ & $4(0.4)$ & $87(12.6)$ & $10(1.5)$ & $0(0)$ \\
\hline Leukopenia & $255(27.4)$ & $113(12.1)$ & $18(1.9)$ & $74(10.7)$ & $21(3.0)$ & $3(0.4)$ \\
\hline Fatigue & $221(23.7)$ & $24(2.6)$ & $3(0.3)$ & $116(16.8)$ & $23(3.3)$ & $\mathrm{I}(0.1)$ \\
\hline Asthenia & $203(21.8)$ & $45(4.8)$ & $0(0)$ & $122(17.7)$ & $27(3.9)$ & $0(0)$ \\
\hline Anemia & 177 (19.0) & $17(1.8)$ & $\mathrm{I}(0.1)$ & $133(19.3)$ & II (I.6) & $2(0.3)$ \\
\hline Pyrexia & $161(17.3)$ & $3(0.3)$ & $0(0)$ & $56(8.1)$ & $4(0.6)$ & $0(0)$ \\
\hline Diarrhea & $158(17.0)$ & $4(0.4)$ & $\mathrm{I}(0.1)$ & $174(25.3)$ & $27(3.9)$ & $\mathrm{I}(0.1)$ \\
\hline Constipation & $154(16.5)$ & $3(0.3)$ & $0(0)$ & 89 (12.9) & $3(0.4)$ & $0(0)$ \\
\hline Headache & $148(15.9)$ & $5(0.5)$ & $0(0)$ & $73(10.6)$ & $0(0)$ & $2(0.3)$ \\
\hline Vomiting & $146(15.7)$ & $5(0.5)$ & $2(0.2)$ & $119(17.3)$ & II (I.6) & $0(0)$ \\
\hline Dyspnea & $129(13.8)$ & $29(3.1)$ & $4(0.4)$ & 79 (II.5) & $19(2.8)$ & $7(1.0)$ \\
\hline Back pain & $124(13.3)$ & $9(1.0)$ & $2(0.2)$ & $49(7.1)$ & $5(0.7)$ & $\mathrm{I}(0.1)$ \\
\hline Weight decreased & $124(13.3)$ & $3(0.3)$ & $0(0)$ & $53(7.7)$ & $\mathrm{I}(0.1)$ & $0(0)$ \\
\hline Cough & $113(12.1)$ & $4(0.4)$ & $0(0)$ & $57(8.3)$ & $\mathrm{I}(0.1)$ & $0(0)$ \\
\hline Arthralgia & $106(11.4)$ & $5(0.5)$ & $\mathrm{I}(0.1)$ & $34(4.9)$ & $3(0.4)$ & $0(0)$ \\
\hline Anorexia & $101(10.8)$ & $2(0.2)$ & $0(0)$ & $32(4.6)$ & $3(0.4)$ & $0(0)$ \\
\hline Bone pain & $97(10.4)$ & $15(1.6)$ & $0(0)$ & $57(8.3)$ & $6(0.9)$ & $0(0)$ \\
\hline Pain in extremity & $94(10.1)$ & $8(0.9)$ & $0(0)$ & $47(6.8)$ & $5(0.7)$ & $0(0)$ \\
\hline Decreased appetite & $73(7.8)$ & $2(0.2)$ & $0(0)$ & $70(10.2)$ & $7(1.0)$ & $0(0)$ \\
\hline ALT increased & $60(6.4)$ & $23(2.5)$ & $0(0)$ & $20(2.9)$ & $2(0.3)$ & $0(0)$ \\
\hline Febrile neutropenia & $32(3.4)$ & $22(2.4)$ & $9(1.0)$ & $9(1.3)$ & $4(0.6)$ & $5(0.7)$ \\
\hline Palmar-plantar erythrodysesthesia syndrome & $8(0.9)$ & $2(0.2)$ & $0(0)$ & $240(34.8)$ & $72(10.4)$ & $0(0)$ \\
\hline
\end{tabular}

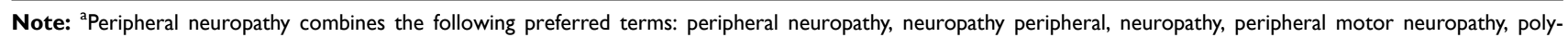
neuropathy, peripheral sensory neuropathy, peripheral sensorimotor neuropathy, demyelinating polyneuropathy, and paraesthesia.

Abbreviations: ALT, alanine transaminase; TEAE, treatment-emergent adverse event.

similar between treatment groups in patients with HER2positive MBC. $^{22}$

EMBRACE and E7389-G000-301 showed that eribulin was generally well-tolerated versus comparator agents (Table 3). ${ }^{17,18}$ In a pooled analysis of data from the Phase III studies, treatment emergent adverse events (TEAEs) occurred in $96.7 \%$ of patients receiving eribulin and $91.3 \%$ of patients in the comparator arms. ${ }^{23}$ The most common Grade 3/4 adverse events (AEs) were neutropenia (45.7\%), leukopenia (14.0\%), peripheral neuropathy (7.3\%), asthenia (4.8\%) and dyspnea (3.5\%) in patients receiving eribulin; and palmar plantar erythrodysesthesia syndrome $(10.4 \%)$, neutropenia $(10.6 \%)$, asthenia $(3.9 \%)$, diarrhea $(4.0 \%)$ and dyspnea $(3.8 \%)$ in those receiving the comparator treatments. Treatment discontinuation due to
TEAEs occurred in $11.3 \%$ of patients receiving eribulin and $13.6 \%$ of those receiving comparator. ${ }^{23}$

\section{Phase III Study of Eribulin in Recurrent/Metastatic Breast Cancer in China}

A Phase III, open-label, randomized study conducted at 35 treatment centers in China found eribulin to be an effective and relatively well-tolerated treatment option for women with previously treated advanced disease (NCT02225470). ${ }^{19}$ This study compared eribulin and vinorelbine in 530 women with locally recurrent/metastatic breast cancer who had received $2-5$ prior chemotherapy regimens, at least two of which were for advanced disease and included an anthracycline and a taxane. Approximately $75 \%$ of 
Events/N

Group/subgroup

Overall

Overall

Receptor status

Her2/neu positive

Her2/neu negative [triple negative]

Her2/neu negative [non-triple negative]

Her2/neu unknown

Prior chemotherapy regimens

2-3

4-5

Age group

$<40$ years

40-64 years

265 years

ECOG performance status

0

1

2

Number of organs involved

$\leq 2$

$>2$

Number of prior chemotherapy regimens

for locally-advanced or metastatic disease

$\leq 2$

$>2$

Prior vinorelbine

No

Yes

Refractory to taxanes

No

Yes

Her2/neu status

Negative

ER status

Negative

Positive

PR status

Negative

Positive

Hormone receptor

Negative

Positive

Triple negative

No

Yes

Eribulin Vinorelbine

Hazard ratio $(95 \% \mathrm{Cl})$

Median (months)

Eribulin Vinorelbine

$203 / 264-181 / 266$

203/264 181/266

$42 / 52 \quad 39 / 52$

$54 / 64 \quad 52 / 68$

98/136 83/131

9/12 7/15

$\begin{array}{ll}112 / 147 & 110 / 150 \\ 91 / 117 & 71 / 116\end{array}$

$30 / 35 \quad 34 / 45$

$163 / 214 \quad 144 / 213$

10/15 $3 / 8$

$39 / 57 \quad 33 / 52$

$154 / 196 \quad 144 / 205$

$10 / 11 \quad 4 / 9$

$74 / 105 \quad 76 / 119$

$129 / 159 \quad 105 / 147$

$121 / 155 \quad 105 / 147$

$82 / 109 \quad 76 / 119$

$169 / 221 \quad 157 / 225$

$34 / 43 \quad 24 / 41$

$71 / 101 \quad 56 / 87$

99/119 103/146

$152 / 200 \quad 135 / 199$

$87 / 109 \quad 87 / 111$

106/148 92/151

113/140 96/127

$84 / 117 \quad 83 / 135$

83/97 $\quad 77 / 97$

$114 / 160 \quad 102 / 165$

$\begin{array}{cc}149 / 200 & 129 / 198 \\ 54 / 64 & 52 / 68\end{array}$
$0.80(0.65,0.98)$

$2.8 \quad 2.8$

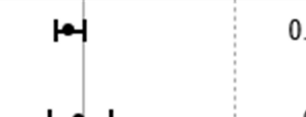

$0.94(0.60,1.48)$

$0.70(0.47,1.03)$

$0.79(0.59,1.06)$

$1.40(0.45,4.39)$

$0.66(0.50,0.87)$

$1.02(0.74,1.41)$

$0.67(0.39,1.17)$

$0.80(0.64,1.02)$

$1.12(0.18,6.80)$

$0.87(0.52,1.43)$

$0.79(0.63,1.01)$

$1.72(0.19,15.60$

$0.73(0.52,1.03)$

$0.87(0.67,1.14)$

$0.69(0.53,0.91)$

$0.91(0.66,1.25)$

$0.76(0.60,0.95)$

$1.01(0.56,1.83)$

$0.76(0.52,1.11)$

$0.88(0.66,1.17)$

$0.76(0.60,0.96)$

$0.79(0.57,10.8)$

$0.78(0.59,1.05)$

$0.74(0.55,0.98)$

$0.80(0.59,1.10)$

$0.75(0.54,1.05)$

$0.79(0.60,1.05)$

4.1

$0.84(0.66,1.06)$

$0.70(0.47,1.03)$

4.0

2.7

2.8

2.7

4.1

2.8

$\begin{array}{ll}3.0 & 2.7 \\ 2.8 & 2.8\end{array}$

\begin{tabular}{ll}
$2.8-2.8$ \\
\hline
\end{tabular}

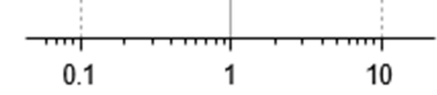

Favours eribulin

Favours vinorelbine

Figure 2 Forest plot of hazard ratio for progression-free survival assessed by independent review, in an intent-to-treat population. Reprinted from Yuan P, Hu X, Sun T, et al. Eribulin mesilate versus vinorelbine in women with locally recurrent or metastatic breast cancer: a randomised clinical trial. Eur j Cancer. 2019;। 12:57-65. Copyright 2019, with permission from Elsevier. ${ }^{19}$

Abbreviations: $\mathrm{Cl}$, confidence interval; ECOG, Eastern Cooperative Oncology Group; ER, estrogen receptor; HER2/neu, human epidermal growth factor receptor 2; HR, hazard ratio; PR, progesterone receptor; ORR, overall response rate. 


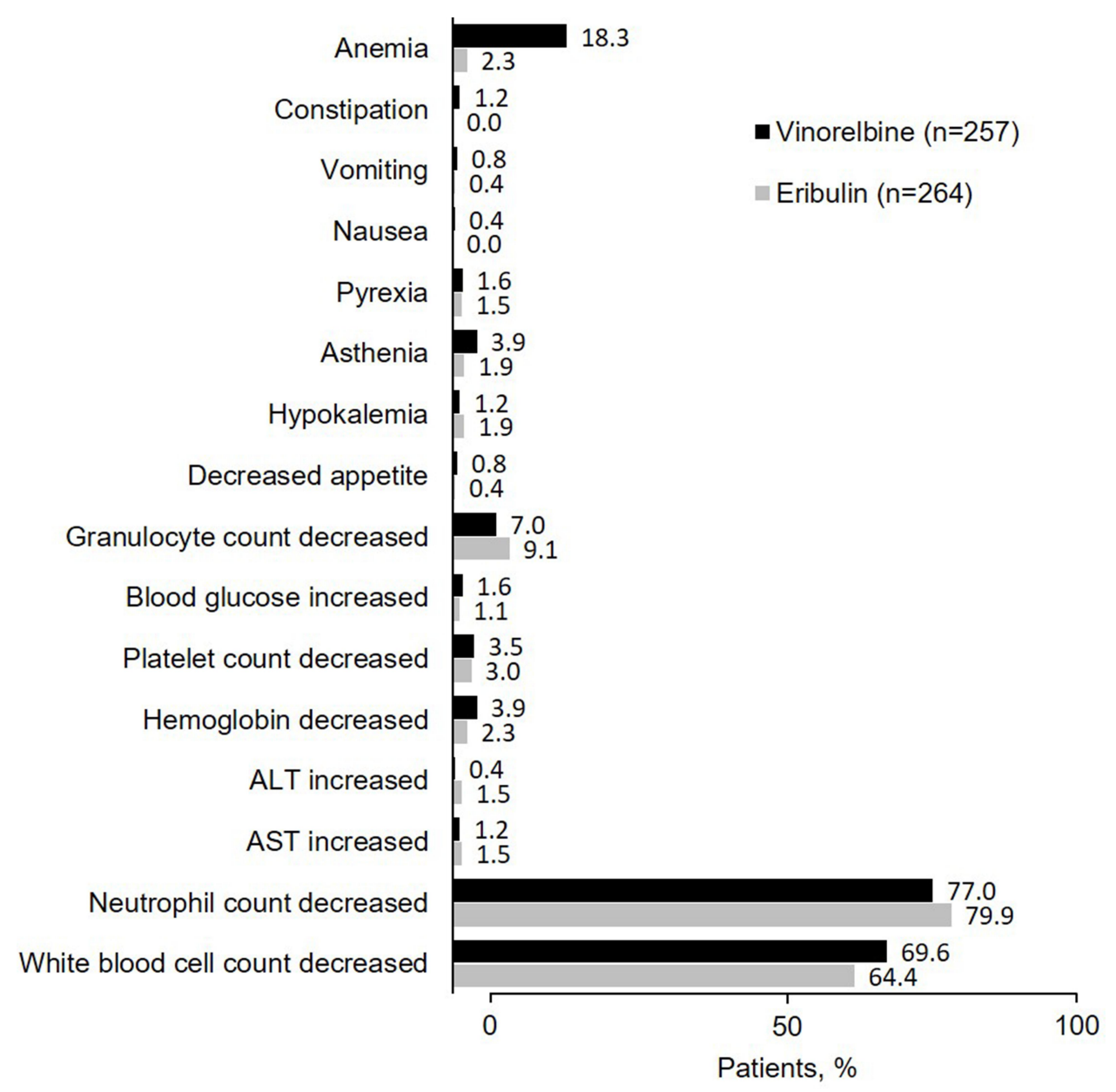

Figure 3 Grade 3/4 treatment emergent adverse events occurring in Chinese patients treated with eribulin or vinorelbine for locally recurrent or metastatic breast cancer. Reproduced from Yuan P, Hu X, Sun T, et al. Eribulin mesilate versus vinorelbine in women with locally recurrent or metastatic breast cancer: a randomised clinical trial. Eur j Cancer. 2019;1 12:57-65. Copyright 2019, with permission from Elsevier. ${ }^{19}$

Abbreviations: ALT, alanine aminotransferase; AST, aspartate aminotransferase.

patients in the study had HER2-negative disease and approximately $25 \%$ had TN disease.

The hazard ratio for progression-free survival (PFS, the primary endpoint) was shown a reduced risk of progression for patients receiving eribulin compared to those receiving vinorelbine, although the median PFS values for the two groups were identical (median PFS: 2.8 vs 2.8 months; hazard ratio [HR], $0.80 ; 95 \% \mathrm{CI}: 0.65-0.98$, $\mathrm{P}=0.036$, Table 1). However, in contrast to the global Phase III studies, no significant difference in OS (a secondary endpoint) was observed between the treatment groups (13.4 months with eribulin vs 12.5 months with vinorelbine $)^{17,18}$ although the objective response rate (ORR) was significantly higher in the eribulin treatment group $(30.7 \%$ vs $16.9 \%$; $\mathrm{P}<0.001)$.

A summary of PFS across various subgroups is presented in Figure 2. Consistent with findings in the international trials, eribulin was associated with improved survival compared to vinorelbine in Chinese patients with HER2negative (HR, 0.76 ; 95\% CI: $0.60-0.96)$ or TN (HR, 0.70; 95\% CI: 0.47-1.03) breast cancer (Table 2).

Eribulin appeared to be better tolerated than vinorelbine, with no new safety signals observed. ${ }^{19}$ TEAEs leading to treatment discontinuation were less frequent with eribulin (7.2\%) than with vinorelbine (14.0\%) as were dose reductions, interruptions, or delays. Grade $\geq 3$ TEAEs, which occurred in $90.2 \%$ of patients treated with eribulin and in $88.3 \%$ of those receiving vinorelbine, were similar between treatment groups and the most common Grade 3/4 TEAEs are summarized in Figure 3. Peripheral neuropathy was uncommon in both treatment arms and no patient receiving eribulin had Grade $\geq 3$ peripheral neuropathy. ${ }^{19}$ A subsequent single-center subgroup analysis of this study including 110 patients reported a significantly higher incidence of autonomic neuropathy among patients who received vinorelbine at weeks 2 and 10 compared with those treated with eribulin. In addition, the 
Table 4 Summary of Real-World Experience with Eribulin in Patients with MBC

\begin{tabular}{|c|c|c|c|}
\hline Study & Patients & Efficacy & Safety \\
\hline $\begin{array}{l}\text { Japanese post- } \\
\text { marketing } \\
\text { observational } \\
\text { study }^{27}\end{array}$ & $\begin{array}{l}95 \mathrm{I} \text { evaluable patients with inoperable or } \\
\text { recurrent breast cancer receiving } I^{\text {st }} \text {-line } \\
\text { eribulin }\end{array}$ & $\begin{array}{l}\text { - ORR: } 16.5 \% \text { (95\% Cl: } 13.7-19.4) \\
\text { - TTF: } 127 \text { days (95\% Cl: } 120-134)\end{array}$ & $\begin{array}{l}\text { Grade } \geq 3 \text { ADRs (>5\% incidence) } \\
\text { - Neutropenia, } 59.8 \% \\
\text { - Leukopenia, } 50.5 \% \\
\text { - Lymphopenia, } 16.1 \% \\
\text { - Febrile neutropenia, } 7.7 \%\end{array}$ \\
\hline $\begin{array}{l}\text { French multi- } \\
\text { center, } \\
\text { retrospective } \\
\text { real-life database }^{28} \\
\text { study }^{28}\end{array}$ & $\begin{array}{l}\text { 16,703 patients with MBC, a proportion of } \\
\text { whom received eribulin as a second- } \\
(n=354) \text {, third- }(647) \text { or fourth-line }(480) \\
\text { treatment }\end{array}$ & $\begin{array}{l}\text { PFS, } 3^{\text {rd }} \text {-line setting } \\
\text { - Eribulin } 4 . I \text { mo } \\
\text { - Other treatments } 3.0 \text { mo } \\
\mathrm{P}=0.001 \\
\text { PFS, } 4^{\text {th }} \text {-line setting } \\
\text { - Eribulin } 3.6 \text { mo } \\
\text { - Other treatments } 2.5 \mathrm{mo} \\
\mathrm{P}=0.010 \\
\text { OS }, 3^{\text {rd }} \text {-line setting } \\
\text { - Eribulin } 11.3 \text { mo } \\
\text { - Other treatments } 7.7 \mathrm{mo} \\
\mathrm{P}<0.001 \\
\text { PFS, } 4^{\text {th }} \text {-line setting } \\
\text { - Eribulin } 10.9 \text { mo } \\
\text { - Other treatments } 6.0 \mathrm{mo} \\
\mathrm{P}<0.001\end{array}$ & \\
\hline $\begin{array}{l}\text { Southeast } \\
\text { Netherlands } \\
\text { Advanced Breast } \\
\text { Cancer Registry }^{29}\end{array}$ & $\begin{array}{l}45 \text { patients received eribulin according to } \\
\text { its registration criteria } \\
45 \text { matched patients eligible for eribulin but } \\
\text { received non-eribulin chemotherapy }\end{array}$ & $\begin{array}{l}\text { Median PFS } \\
\text { - Eribulin: } 3.5 \text { mo (95\% Cl: } 2.7-5.5) \\
\text { - Non-eribulin: } 3.2 \text { mo }(95 \% \text { Cl: } \\
\text { 2.0-4.8) } \\
\text { Adjusted HR: } 0.83,95 \% \\
\text { CI: } 0.49-1.38 \text {. } \\
\text { Median OS } \\
\text { - Eribulin: } 5.9 \text { mo (95\% CI: } \\
\text { 4.6-11.0) } \\
\text { - Non-eribulin: } 5.2 \text { mo (95\% } \\
\text { CI: 4.6-9.5) } \\
\text { Adjusted HR: } 0.66,95 \% \\
\text { CI: } 0.38-1.13 \text {. }\end{array}$ & $\begin{array}{l}\text { - } 31 \% \text { of patients receiving eribulin } \\
\text { required dose delay or reduction. } \\
\text { - Leukopenia and neutropenia were } \\
\text { the most common reason leading } \\
\text { to delay or reduction in dose }(n=6 \text {, } \\
\text { I3\% each) }\end{array}$ \\
\hline
\end{tabular}

Abbreviations: ADR, adverse drug reaction; HR, hazard ratio; MBC, metastatic breast cancer; mo, months; ORR, objective response rate; OS, overall survival; PFS, progression-free survival; TTF, time to treatment failure.

onset of neurotoxicity appeared to occur earlier with vinorelbine compared with eribulin. ${ }^{20}$

The findings of this trial supported the recent approval of eribulin in China in 2019. ${ }^{26}$ The approved clinical indication for eribulin in China is for patients with locally advanced/metastatic breast cancer after treatment failure with at least two chemotherapy regimens, including an anthracycline and a taxane.

\section{Eribulin in a Real-World Treatment Setting}

Further data on the real-world clinical efficacy and safety of eribulin in Asian patients are available from a Japanese post-marketing observational study. ${ }^{27}$ This study included 951 evaluable patients with inoperable or recurrent breast cancer receiving eribulin in the first-line treatment setting. Approximately $74 \%$ of patients had HER2-negative disease and $18 \%$ had TN disease. The ORR was $16.5 \%$ (95\% CI: 13.7-19.4), which is similar to the rates achieved in the international studies but lower than those achieved in the Chinese Phase III study (ORR, 30.7\%).

The incidence of adverse drug reactions (ADRs) in the Japanese post-marketing study was $88.4 \%$, and the most common events (neutropenia, leukopenia, and lymphopenia) were similar to the most common AEs reported in the Phase III trials (Table 4). 
Additional real-world evidence with eribulin has been derived from the national observational ESME program, a French multi-center, retrospective real-life database incorporating data from 18 French Comprehensive Cancer Centers. ${ }^{28}$ This real-life study included a total of 16,703 patients with $\mathrm{MBC}$, a proportion of whom received eribulin as a second- $(\mathrm{n}=354)$, third- (647) or fourth-line (480) treatment. In the second-line setting, there was no significant difference in PFS between eribulin and other chemotherapies; however, PFS and OS were significantly longer in patients receiving eribulin in the third- and fourth-line settings compared to treatment with "other chemotherapies (Table 4)." In a subgroup analysis of patients with HER2-negative disease in the second-line setting, both PFS and OS were significantly improved among those patients receiving eribulin compared to "other treatments" (PFS: 4.6 months vs 3.9 months, $\mathrm{P}=0.038$; OS: 15.0 months vs 10.5 months, $\mathrm{P}=0.011){ }^{28}$

A registry study from the Netherlands Advanced Breast Cancer Group has compared clinical outcomes in 45 patients with advanced breast cancer who received eribulin and 45 matched patients who were eligible for eribulin therapy but who received a different chemotherapy (most frequently capecitabine, vinorelbine, or nonpegylated liposomal doxorubicin). ${ }^{29}$ There was no difference in PFS or OS between these populations, and eribulin was considered to have a manageable toxicity profile (Table 2). On multivariate analyses, compared to patients with HER2-positive disease (the reference group), the presence of triple-negative disease and CNS metastases was significantly associated with reduced PFS and OS. These data suggest that eribulin may be considered as a treatment option for patients with advanced breast cancer.

\section{Efficacy of Eribulin in Specific Breast Cancer Subtypes}

Preclinical and mechanistic studies indicate that eribulin may provide a clinical benefit for specific patient groups, including those with disease resistant to taxane therapy. Data from Phase III trials in patients who had received prior anthracycline and taxane therapy support the use of eribulin in this patient population, as do Phase II studies with eribulin alone or in combination with other agents in the first-and later-line settings. The results of studies in patients with HER2-positive, HER2-negative and TN disease are summarized in Table 2.

\section{Eribulin in HER2-Positive Metastatic Breast Cancer}

Phase II trials in patients with HER2-positive, locally recurrent or metastatic breast cancer have shown that firstline combination therapy with eribulin plus trastuzumab achieves a high ORR (54-80\%) (Table 2). ${ }^{30-33}$ In a prespecified analysis of one of these studies, prior trastuzumab use in the neoadjuvant or adjuvant setting did not appear to affect the response to eribulin plus trastuzumab in patients with metastatic disease. ${ }^{32}$ However, outcomes of eribulin therapy (ORR and PFS) were generally poorer in patients who had received prior anthracycline and/or taxane therapy than in those who were anthracycline- and taxane-naïve. ${ }^{31}$ Overall, the combination of eribulin with anti-HER2 therapies was generally well tolerated. The outcomes from several other Phase II studies in patients with HER2-positive breast cancer are summarized in Table 2. ${ }^{34,35}$ When comparing these outcomes in HER2positive patients with the results from the Global and Chinese Phase III trials of eribulin, it should be noted that patients with HER2-positive disease included in the Phase III trials did not receive HER2-targeted therapy. ${ }^{17-19}$ The combination of eribulin with pertuzumab and trastuzumab is also being compared with paclitaxel or docetaxel plus pertuzumab/trastuzumab in an ongoing Phase III trial in the first-line treatment setting (patients could have received prior ado-trastuzumabemtansine; NCT03264547). ${ }^{36}$

\section{Eribulin in HER2-Negative Metastatic Breast Cancer}

The data summarized in the following sections refer to patients who were HER2- but not triple negative.

\section{Neo-Adjuvant Treatment}

The clinical benefit of eribulin in the neoadjuvant setting in patients with HER2-negative, locally advanced breast cancer has been evaluated in Phase II feasibility studies. ${ }^{37,38}$ Neoadjuvant eribulin followed by doxorubicin and cyclophosphamide (AC) was compared with weekly paclitaxel in patients with HER2-negative locally advanced breast cancer. Patients were randomized (1:2) to receive either paclitaxel $(n=19)$ for 12 treatments or eribulin $(n=31)$ every 3 weeks for 4 cycles followed by AC every 3 weeks for 4 cycles before surgery. Although eribulin was well tolerated in this treatment setting, the pathological compete response (pCR) rate was similar to 
that achieved with weekly paclitaxel $(17 \%$ vs $26 \%){ }^{37}$ Another randomized Phase II study compared neoadjuvant eribulin/cyclophosphamide versus docetaxel/cyclophosphamide in women with operable HER2-negative breast cancer. Patients were randomized 2:1 to receive either eribulin on days 1 and 8 plus cyclophosphamide on day $1(n=44)$ or docetaxel plus cyclophosphamide on day 1 $(n=22)$ administered every 21 days for 6 cycles, followed by surgery. Both regimens produced similar pCR rates ( $13 \%$ and $9 \%$, respectively) with no difference in efficacy between the two regimens, and there was no evidence of improved efficacy with eribulin/cyclophosphamide versus docetaxel/cyclophosphamide. ${ }^{38}$

In on-going studies in the neoadjuvant treatment setting, eribulin with sequential 5-fluorouracil/doxorubicin/ cyclophosphamide is being compared with paclitaxel plus sequential 5-fluorouracil/epirubicin/cyclophosphamide in patients with HER2-negative early-stage breast cancer (NCT01593020), eribulin followed by doxorubicin/cyclophosphamide is being evaluated in patients with HER2negative inflammatory breast cancer (NCT02623972), and eribulin plus carboplatin is being investigated in patients with stage I-III TNBC (NCT01372579). The findings of these trials will help to more clearly determine if eribulin has a role to play in the pre-operative treatment setting for women with operable breast cancer.

\section{Eribulin as First-Line Systemic Treatment}

Eribulin has shown promising activity as a first-line therapy in patients with HER2-negative MBC in Phase II studies, with ORRs ranging between approximately $20 \%$ and 54\%, and with median PFS/time-to-progression (TTP) ranging from 4.1 to 6.8 months (Table 2). ${ }^{31,39-43}$ One of these studies included a prespecified analysis of efficacy by prior anthracycline and/or taxane use and found that eribulin had similar clinical activity in patients who were chemotherapy-naïve and those who had received prior therapy. $^{31}$ ORR and median PFS were $25.9 \%$ and 5.8 months among patients who had received prior anthracycline, $26.9 \%$ and 5.8 months among patients who had received prior taxane, $25.0 \%$ and 6.7 months among patients who had received both anthracycline and taxane, and $30.4 \%$ and 7.6 months among patients who were anthracycline- and taxane-naïve. ${ }^{31}$

A Phase II study has assessed treatment with eribulin alone or in combination with pembrolizumab in 88 patients with hormone receptor positive, HER2-negative MBC. ${ }^{44}$ Patients were required to have received $\geq 2$ lines of hormonal therapy and $\leq 2$ lines of chemotherapy. Median PFS was 4.1 months and 4.2 months in the combination and monotherapy arms (HR, 0.80; 95\% CI: 0.$50-1.26 ; \mathrm{P}=0.30$ ) and the ORR was $27 \%$ versus $34 \%$, respectively. In this study, the addition of pembrolizumab to eribulin did not extend PFS or improve ORR. ${ }^{44}$

\section{Eribulin as Later-Line Systemic Treatment}

Subgroup and pooled analyses of the Phase III trials of eribulin suggest that eribulin treatment leads to significantly better survival outcomes than comparator agents (TPC, capecitabine or vinorelbine) in patients with HER2-negative disease. ${ }^{19,21,22,25}$ For example, patients with HER2-negative disease in the Chinese Phase III study $(n=399)$ had improved PFS with eribulin compared with vinorelbine. ${ }^{19}$ Prolonged survival with eribulin was also seen in this patient subgroup in a Japanese realworld analysis; median OS was 15.6 months in 637 patients with HER2-negative disease receiving eribulin as first-, second-, third-, or later-line therapy. ${ }^{45}$ In addition, results from a Phase I trial of combination therapy with eribulin plus balixafortide in 54 patients with heavily pretreated HER2-negative disease showed promising results with an ORR of $30 \%{ }^{46}$ An ongoing Phase III trial is further investigating this combination in HER2negative patients with locally recurrent or metastatic breast cancer (NCT03786094).

\section{Eribulin in Triple-Negative Metastatic Breast Cancer}

Subgroup analyses of patients with TN MBC included in the international and Chinese Phase III studies of eribulin showed that patients who received eribulin had significantly better survival outcomes than those assigned to comparator agents including TPC, capecitabine or vinorelbine. ${ }^{19,21,22}$ Approximately $23 \%$ of patients enrolled in the international Phase III clinical trials had $\mathrm{TN}$ disease (eribulin, $\mathrm{n}=243$ [22.9\%]; control, $\mathrm{n}=185$ [23.1\%]). ${ }^{22}$ In a pooled analysis of these two trials, eribulin was associated with significantly improved OS versus comparator (12.9 months vs 8.2 months; $\mathrm{P}=0.006$ ). The subgroup analysis of the Chinese trial, which included 132 patients with TN disease, also showed that PFS was improved with eribulin versus vinorelbine (HR, 0.70; 95\% CI: 0.47-1.03).

Data from prospective clinical trials of eribulin in patients with TN disease are limited at present. Two Phase I/II trials that compared eribulin in combination with either everolimus or olaparib have shown promising results, and 
trials of other treatment combinations are ongoing (Table 4). In a Phase I study including 25 patients with heavily pretreated TN breast cancer, eribulin plus everolimus showed modest activity (partial response rate, 36\%; TTP, 2.6 months; median OS, 8.3 months). ${ }^{47}$ A Phase I/II trial of eribulin plus olaparib that included 24 Japanese patients with TN breast cancer previously treated with anthracyclines and taxanes reported a response rate of $29.2 \%$, and median PFS and OS of 4.2 and 14.5 months, respectively. ${ }^{48}$

\section{Predictors of Eribulin Efficacy}

Various studies have sought to identify predictors of eribulin efficacy in patients with MBC. Tumor infiltrating lymphocytes (TILs) were identified as an independent indicator of outcome in patients with TNBC. ${ }^{49}$ Patients with higher numbers of TILs had significantly longer PFS than patients with lower TIL numbers. Neutrophil-tolymphocyte ratio (NLR), a marker of systemic immunity, was significantly associated with PFS in eribulin-treated patients but not in those treated with nab-paclitaxel in a single-institute retrospective study in patients with MBC. ${ }^{50}$ Median PFS in patients with a baseline NLR $<3$ was significantly longer than PFS in patients with a baseline NLR $\geq 3$ (242 days vs 98 days; HR, 0.37; 95\% CI: 0.18-0.71). High absolute lymphocyte counts (ALC) have been associated with prolonged survival in patients with MBC treated with eribulin, but not TPC in patients enrolled in the EMBRACE trial. ${ }^{51}$ OS was prolonged with eribulin vs TPC in patients with baseline ALC $\geq 1500 / \mu \mathrm{L}$ (HR, 0.59; 95\% CI: 0.437-0.784; P<0.001). However, there was no significant difference by treatment in patients with $\mathrm{ALC}<1500 / \mu \mathrm{L}$.

\section{Guideline Updates and Current Treatment Approaches in China}

The improved survival outcomes and tolerability of eribulin compared with vinorelbine reported by the Chinese Phase III trial have supported the first approval of eribulin in China for women with locally advanced/metastatic breast cancer. In addition, in the most recent Chinese Society of Clinical Oncology (CSCO) breast cancer guidelines, eribulin has been given a Grade $2 \mathrm{~B}$ recommendation as first-line rescue therapy for patients with HER2negative disease after treatment failure with anthracyclines and paclitaxel. In addition, the Chinese Anti-Cancer Association Breast Cancer Diagnosis and Treatment Guidelines (2019) cites eribulin as a commonly used single agent chemotherapy for recurrent/metastatic breast cancer. $^{52}$ Other single agents recommended in the same setting include capecitabine, vinorelbine, gemcitabine (all Grade 2A) albumin-bound paclitaxel and etoposide (both Grade $2 \mathrm{~B}){ }^{53}$

Several Phase III trials of eribulin have shown that patients with HER2-negative breast cancer respond well to eribulin, with significantly improved survival outcomes versus TPC and vinorelbine. These trials included patients whose disease had progressed after prior anthracycline and taxane therapy, suggesting that eribulin can bypass

Table 5 Ongoing Phase II and III Studies of Eribulin Combination Therapy in Patients with Locally Advanced/Metastatic Breast Cancer (ClinicalTrials.gov, Accessed July 2, 2020)

\begin{tabular}{|c|c|c|}
\hline & $\begin{array}{l}\text { Trial Phase } \\
\text { (Primary } \\
\text { Completion } \\
\text { Date) }\end{array}$ & $\begin{array}{l}\text { Patient Group } \\
\text { (ClinicalTrials.gov } \\
\text { ID) }\end{array}$ \\
\hline $\begin{array}{l}\text { Eribulin }+ \\
\text { balixafortide }\end{array}$ & 3 (March 202I) & $\begin{array}{l}\text { HER2-negative locally } \\
\text { recurrent/MBC } \\
\text { (NCT03786094) }\end{array}$ \\
\hline $\begin{array}{l}\text { Eribulin }+ \\
\text { pertuzumab + } \\
\text { trastuzumab vs } \\
\text { paclitaxel/docetaxel + } \\
\text { pertuzumab + } \\
\text { trastuzumab }\end{array}$ & 3 (April 2022) & $\begin{array}{l}\text { HER2-positive } \\
\text { advanced/recurrent BC } \\
\text { (no prior history of } \\
\text { chemotherapy except } \\
\text { ado-trastuzumab } \\
\text { emtansine; } \\
\text { NCT03264547) }\end{array}$ \\
\hline $\begin{array}{l}\text { Eribulin + } \\
\text { pembrolizumab }\end{array}$ & 2 (August 2020) & $\begin{array}{l}\text { HR-positive MBC } \\
\text { (NCT03051659) }\end{array}$ \\
\hline $\begin{array}{l}\text { Eribulin + } \\
\text { pembrolizumab }\end{array}$ & $\begin{array}{l}2 \text { (October 2018/ } \\
2020)\end{array}$ & $\begin{array}{l}\text { HR-positive/HER2- } \\
\text { negative MBC } \\
\text { (NCT03222856) }\end{array}$ \\
\hline $\begin{array}{l}\text { Eribulin + endocrine } \\
\text { therapy }\end{array}$ & 2 (May 202I) & $\begin{array}{l}\text { Locally recurrent/ } \\
\text { metastatic BC } \\
\text { (NCT037950I2) }\end{array}$ \\
\hline Eribulin + nivolumab & 2 (Sept 2020) & $\begin{array}{l}\text { HER2-negative MBC } \\
\text { (NCT04061863) }\end{array}$ \\
\hline $\begin{array}{l}\text { Eribulin }+ \\
\text { atezolizumab + } \\
\text { cobimetinib }\end{array}$ & 2 (August 2020) & $\begin{array}{l}\text { Recurrent/metastatic } \\
\text { inflammatory BC } \\
\text { (NCT032023I6) }\end{array}$ \\
\hline $\begin{array}{l}\text { Camrelizumab + } \\
\text { apatinib + eribulin }\end{array}$ & $\begin{array}{l}2 \\
\text { (December 2022) }\end{array}$ & $\begin{array}{l}\text { Advanced TN BC } \\
\text { (NCT0430374I) }\end{array}$ \\
\hline Eribulin + copanlisib & I/2 (March 202I) & $\begin{array}{l}\text { Metastatic TN BC } \\
\text { (NCT043459/3) }\end{array}$ \\
\hline
\end{tabular}

Abbreviations: BC, Breast cancer; HER2, human epidermal growth factor receptor 2; HR, hormone receptor; $M B C$, metastatic breast cancer; $T N$, triple negative. 
resistance mechanisms that prevent activity with other agents. These findings align with preclinical studies indicating that eribulin has a mechanism of action distinct from other microtubule inhibitors such as taxanes and vinca alkaloids. In the future, the unique mechanism of action of eribulin coupled with its clinical efficacy/safety profile may permit wider use of this agent in patients who would otherwise be considered for standard taxane treatment. In addition, there are multiple on-going Phase II and III studies investigating eribulin combination regimens, and these are summarized in Table 5.

Decisions regarding treatment with eribulin should take account of the toxicity profiles of approved agents. Eribulin appeared to be better tolerated than vinorelbine in the Chinese Phase III trial. ${ }^{19}$ In particular, TEAEs leading to treatment discontinuation were less frequent with eribulin compared with vinorelbine, as were dose reductions, interruptions, or delays.

Peripheral neuropathy is the most common doselimiting side effect associated with microtubule-targeted agents. However, preclinical ${ }^{54-56}$ and clinical data support an improved profile of peripheral neuropathy with eribulin. Studies conducted in mice have shown that eribulin has less neurotoxic impact compared to paclitaxel and ixabepilone. $^{54-56}$ In a comparative Phase II study in patients with $\mathrm{MBC}$, time to onset of neuropathy was 35.9 weeks with eribulin and 11.6 weeks with ixabepilone; and time to resolution was 48 versus 10 weeks, respectively. In addition, the incidence of neuropathy and peripheral neuropathy associated with eribulin and ixabepilone were $33.3 \%$ versus $48.0 \%$ and $31.4 \%$ versus $44.0 \%$, respectively $(\mathrm{P}=\mathrm{NS}){ }^{57}$ Furthermore, peripheral neuropathy was uncommon among patients receiving eribulin in the Chinese Phase III study, ${ }^{19}$ and vinorelbine was associated with a significantly higher incidence of autonomic neuropathy compared with eribulin, and was also associated with an earlier onset of neurotoxicity in a subgroup analysis from this study. ${ }^{20}$ A randomized, multicenter trial including 118 patients with HER2negative $\mathrm{MBC}$ showed that the incidence of neurotoxicity associated with eribulin was significantly lower than for paclitaxel (both given in combination with gemcitabine). In this study, the incidence of Grade $\geq 2$ neurotoxicity was $13.6 \%$ with the eribulin regimen versus $45.8 \%$ for paclitaxel $(\mathrm{P}<0.001) .{ }^{43}$ The ongoing Phase IV PAINTER study will evaluate the safety profile of eribulin in patients with MBC, with a particular focus on identifying patients at risk of developing neuropathy. An association with a single nucleotide polymorphism and toxicity is being investigated, and results are awaited with interest (NCT02864030).

\section{Conclusions and Future Directions}

The need for effective treatment options for women diagnosed with $\mathrm{MBC}$ in China remains unmet. Currently available therapies are largely palliative and the 5-year OS rates for patients with stage I, II, III, and IV diseases are $96.5 \%$, $91.6 \%, 74.8 \%$, and $40.7 \%{ }^{58}$ Improving outcomes for patients with different subtypes of MBC using novel strategies that prolong survival and enhance quality of life after a diagnosis of metastatic disease is critical.

Eribulin is a new addition to the treatment armamentarium for MBC in China. It was approved in 2019 for patients with locally advanced/metastatic breast cancer after treatment failure with at least two chemotherapy regimens, including an anthracycline and a taxane. In the Phase III EMBRACE study, eribulin was found to improve OS compared with treatment of physicians' choice in patients with locally recurrent/metastatic disease that had progressed following prior taxane and anthracycline therapy. Its efficacy in the Asian population was subsequently confirmed in a Phase III clinical trial conducted in China ${ }^{19}$ and in the real-world treatment setting in Japan. ${ }^{27}$ Consistent with findings from international studies, survival was better in patients in China with HER2-negative or $\mathrm{TN}$ breast cancer who received eribulin than in those given vinorelbine. $^{19}$ Evidence Blocks from the National Comprehensive Cancer Network (NCCN) for 2020 provide a visual representation of the key measures of oncology therapies, allowing health care providers and patients to make informed decisions regarding treatment choices. ${ }^{59,60}$ The evidence block scores for eribulin are $4 / 5$ for efficacy, indicating that the agent is considered very effective (cure unlikely but sometimes provides a long-term survival advantage), similar to the efficacy evidence scores for doxorubicin and paclitaxel. Coupled with clinical activity, the improved safety and tolerance of eribulin compared with vinorelbine and other agents (described above) indicate that eribulin may be a preferred option for use after taxanes and anthracyclines.

In China, combination therapy is more widely used than single agent therapy in the MBC treatment setting. Agents such as eribulin that have well-characterized and favorable tolerability profiles are advantageous in combination therapy regimens. Preliminary data from Phase II studies indicate that eribulin may be safely combined with 
various agents including everolimus or olaparib in patients with TNBC, ${ }^{47,48}$ and gemcitabine in patients with HER2negative $\mathrm{MBC}{ }^{43}$ Indeed, eribulin/gemcitabine combination therapy was associated with significantly less neurotoxicity versus taxane/gemcitabine in a randomized, multicenter trial in patients with HER2-negative breast cancer. $^{43}$ Eribulin is also being investigated as part of a triple therapy regimen with trastuzumab and pertuzumab in an on-going Phase III trial in the first-line treatment setting in patients with HER2-positive disease (NCT03264547). Other investigational eribulin combinations include nivolumab, pembrolizumab and atezolizumab (Table 5). The efficacy and safety of eribulin in combination with camrelizumab + apatinib in patients with TNBC is currently being investigated in a Chinese study (NCT04303741).

It is hoped that the findings from these on-going trials will help to direct approaches to combination therapy with eribulin, and will lead to improved clinical efficacy and a good safety profile. Predictors of clinical response to eribulin that have been identified so far include TILs, NLR, and ALC. On-going research in China is also focusing on identifying predictors of response to eribulin in Chinese patients with MBC.

Overall, eribulin appears to be a very promising drug that carries hope for millions of patients with breast cancer in China, especially those with late-stage metastasis, and across the various MBC disease subtypes including HER2negative, HER2-positive, and TN disease. Expanded combination treatment strategies incorporating eribulin may provide increased opportunities for prolonged survival and improved patient quality of life.

\section{Funding}

No funding was received for the writing of this article.

\section{Disclosure}

Binghe $\mathrm{Xu}$ reports grants from Hengrui, personal fees from Novartis, advisory fees from Roche, and fees for serving on speakers' bureau from AstraZeneca, Pfizer, Eisai, and Roche, during the conduct of the study. The authors have no other potential conflicts of interest to declare for this work.

\section{References}

1. Chen W, Zheng R, Baade PD, et al. Cancer statistics in China, 2015. CA Cancer J Clin. 2016;66(2):115-132. doi:10.3322/caac.21338
2. Sung H, Rosenberg PS, Chen W-Q, et al. Female breast cancer incidence among Asian and Western populations: more similar than expected. JNCI. 2015;107(7):7. doi:10.1093/jnci/djv107

3. Cheng Y, Yan Y, Gong J, Yang N, Nie S. Trends in incidence and mortality of female breast cancer during transition in Central China. Cancer Manage Res. 2018;10:6247-6255. doi:10.2147/CMAR.S182510

4. National Cancer Institute. Surveillance, Epidemiology and End Results Programme (SEER); 2020; Available from: https://seer.can cer.gov/. Accessed October 1, 2020.

5. National Comprehensive Cancer Network. NCCN Clinical Practice Guidelines in Oncology: breast Cancer v4.2020; 2020. Available from: https://www.ncen.org/professionals/physician_gls/pdf/breast.pdf. Accessed October 1, 2020.

6. Towle MJ, Nomoto K, Asano M, Kishi Y, Yu MJ, Littlefield BA. Broad spectrum preclinical antitumor activity of eribulin (Halaven(R)): optimal effectiveness under intermittent dosing conditions. Anticancer Res. 2012;32(5):1611-1619.

7. Towle MJ, Salvato KA, Budrow J, et al. In vitro and in vivo anticancer activities of synthetic macrocyclic ketone analogues of halichondrin B. Cancer Res. 2001;61(3):1013-1021.

8. Kuznetsov G, TenDyke K, Yu M, Littlefield BA. Antiproliferative Effects of Halichondrin B Analog Eribulin Mesylate (E7389) Against Paclitaxel Resistant Human Cancer Cells in Vitro. Paper Presented At: AACR-NCI-EORTC International Conference: Molecular Targets and Cancer Therapeutics. San Francisco; 2007.

9. Jordan MA, Wilson L. Microtubules as a target for anticancer drugs. Nat Rev Cancer. 2004;4(4):253-265. doi:10.1038/nrc1317

10. Towle MJ, Salvato KA, Wels BF, et al. Eribulin induces irreversible mitotic blockade: implications of cell-based pharmacodynamics for in vivo efficacy under intermittent dosing conditions. Cancer Res. 2011;71(2):496-505. doi:10.1158/0008-5472.CAN-101874

11. Jordan MA, Kamath K, Manna T, et al. The primary antimitotic mechanism of action of the synthetic halichondrin E7389 is suppression of microtubule growth. Mol Cancer Ther. 2005;4(7):1086-1095. doi:10.1158/1535-7163.MCT-04-0345

12. Alday PH, Correia JJ. Macromolecular interaction of halichondrin B analogues eribulin (E7389) and ER-076349 with tubulin by analytical ultracentrifugation. Biochemistry. 2009;48(33):7927-7938. doi:10.1021/ bi900776u

13. Smith JA, Wilson L, Azarenko O, et al. Eribulin binds at microtubule ends to a single site on tubulin to suppress dynamic instability. Biochemistry. 2010;49(6):1331-1337. doi:10.1021/ bi901810u

14. Kuznetsov G, Towle MJ, Cheng H, et al. Induction of morphological and biochemical apoptosis following prolonged mitotic blockage by halichondrin B macrocyclic ketone analog E7389. Cancer Res. 2004;64(16):5760-5766. doi:10.1158/0008-5472.CAN-04-1169

15. Agoulnik S, Kuznetsov G, TenDyke K, Parent LA, Marsh JP, Twine N. Sensitivity to halichondrin analog E7389 and hemiasterlin analog E7974 correlates with $\beta \mathrm{III}$ tubulin isotype expression in human breast cancer cell lines. $J$ clin oncol. 2005;23(16_suppl):2012. doi:10.1200/ jco.2005.23.16_suppl.2012

16. Funahashi Y, Okamoto K, Adachi Y, et al. Eribulin mesylate reduces tumor microenvironment abnormality by vascular remodeling in preclinical human breast cancer models. Cancer Sci. 2014;105 (10):1334-1342. doi:10.1111/cas.12488

17. Cortes J, O’Shaughnessy J, Loesch D, et al. Eribulin monotherapy versus treatment of physician's choice in patients with metastatic breast cancer (EMBRACE): a phase 3 open-label randomised study. Lancet. 2011;377(9769):914-923. doi:10.1016/S0140-6736(11)60070-6

18. Kaufman PA, Awada A, Twelves C, et al. Phase III open-label randomized study of eribulin mesylate versus capecitabine in patients with locally advanced or metastatic breast cancer previously treated with an anthracycline and a taxane. J clin oncol. 2015;33(6):594-601. doi:10.1200/JCO.2013.52.4892 
19. Yuan P, Hu X, Sun T, et al. Eribulin mesilate versus vinorelbine in women with locally recurrent or metastatic breast cancer: a randomised clinical trial. Eur j Cancer. 2019;112:57-65. doi:10.1016/j.ejca.2019.02.002

20. Wu Y, Wang Q, Zhang J, Cao J, Wang B, Hu X. Incidence of peripheral neuropathy associated with eribulin mesylate versus vinorelbine in patients with metastatic breast cancer: sub-group analysis of a randomized phase III study. Support Care Cancer. 2020;28 (8):3819-3829. doi:10.1007/s00520-019-05112-5

21. Twelves C, Awada A, Cortes J, et al. Subgroup analyses from a phase 3, open-label, randomized study of eribulin mesylate versus capecitabine in pretreated patients with advanced or metastatic breast cancer. Breast Cancer (Auckl). 2016;10:77-84. doi:10.4137/BCBCR.S39615

22. Twelves C, Cortes J, Vahdat L, et al. Efficacy of eribulin in women with metastatic breast cancer: a pooled analysis of two phase 3 studies. Breast Cancer Res Treat. 2014;148(3):553-561. doi:10.1007/s10549-014-3144-y

23. Pivot X, Marme F, Koenigsberg R, Guo M, Berrak E, Wolfer A Pooled analyses of eribulin in metastatic breast cancer patients with at least one prior chemotherapy. Ann Oncol. 2016;27(8):1525-1531. doi:10.1093/annonc/mdw203

24. Cortes J, Twelves C. Impact of the number of prior chemotherapy regimens on outcomes for patients with metastatic breast cancer treated with eribulin: a post hoc pooled analysis. Breast J. 2020;26 (7):1347-1351. doi:10.1111/tbj.13686

25. Pivot X, Im SA, Guo M, Marme F. Subgroup analysis of patients with HER2-negative metastatic breast cancer in the second-line setting from a phase 3, open-label, randomized study of eribulin mesilate versus capecitabine. Breast Cancer. 2018;25(3):370-374. doi:10.1007/s12282017-0826-4

26. Eisai CL Anticancer agent halaven ${ }^{\circledR}$ approved for treatment of locally advanced or metastatic breast cancer in China. 2019; Available from: https://www.eisai.com/news/2019/news201952.html. Accessed October $1,2020$.

27. Watanabe J, Ito Y, Ohsumi S, et al. Safety and effectiveness of eribulin in Japanese patients with locally advanced or metastatic breast cancer: a post-marketing observational study. Invest New Drugs. 2017;35(6):791-799. doi:10.1007/s10637-017-0486-4

28. Jacot W, Heudel P-E, Fraisse J, et al. Real-life activity of eribulin mesylate among metastatic breast cancer patients in the multicenter national observational ESME program. Int $j$ Cancer. 2019;145(12):3359-3369. doi:10.1002/ijc.32402

29. Pouwels X, Geurts SME, Ramaekers BLT, et al. The relative effectiveness of eribulin for advanced breast cancer treatment: a study of the southeast Netherlands advanced breast cancer registry. Acta Oncol. 2020;59(1):82-89. doi:10.1080/0284186X.2019.1670356

30. Wilks S, Puhalla S, O’Shaughnessy J, et al. Phase 2, multicenter, single-arm study of eribulin mesylate with trastuzumab as first-line therapy for locally recurrent or metastatic HER2-positive breast cancer. Clin Breast Cancer. 2014;14(6):405-412. doi:10.1016/j. clbc.2014.04.004

31. O'Shaughnessy J, McIntyre K, Schwartzberg L, et al. Impact of prior anthracycline or taxane use on eribulin effectiveness as first-line treatment for metastatic breast cancer: results from two phase 2, multicenter, single-arm studies. SpringerPlus. 2015;4 (1):532. doi:10.1186/s40064-015-1322-y

32. Puhalla S, Wilks S, Brufsky AM, et al. Clinical effects of prior trastuzumab on combination eribulin mesylate plus trastuzumab as first-line treatment for human epidermal growth factor receptor 2 positive locally recurrent or metastatic breast cancer: results from a Phase II, single-arm, multicenter study. Breast Cancer. 2016;8:231-239. doi:10.2147/BCTT.S98696

33. Sakaguchi K, Nakatsukasa K, Koyama H, et al. Phase II clinical trial of first-line eribulin plus trastuzumab for advanced or recurrent HER2-positive breast cancer. Anticancer Res. 2018;38 (7):4073-4081. doi:10.21873/anticanres.12697
34. Inoue $\mathrm{K}$, Ninomiya $\mathrm{J}$, Saito $\mathrm{T}$, et al. Eribulin, trastuzumab, and pertuzumab as first-line therapy for patients with HER2-positive metastatic breast cancer: a phase II, multicenter, collaborative, open-label, single-arm clinical trial. Invest New Drugs. 2019;37 (3):538-547. doi:10.1007/s10637-019-00755-x

35. Yamashita T, Kawaguchi H, Masuda N, et al. Efficacy of the eribulin, pertuzumab, and trastuzumab combination therapy for human epidermal growth factor receptor 2-positive advanced or metastatic breast cancer: a multicenter, single arm, phase II study (JBCRG-M03 study). Invest New Drugs. 2020. doi:10.1007/s10637-020-00991-6

36. Yamashita T, Masuda N, Saji S, et al. Trastuzumab, pertuzumab, and eribulin mesylate versus trastuzumab, pertuzumab, and a taxane as a first-line or second-line treatment for HER2-positive, locally advanced or metastatic breast cancer: study protocol for a randomized controlled, non-inferiority, phase III trial in Japan (JBCRG-M06/EMERALD). Trials. 2020;21(1):391.

37. Abraham J, Robidoux A, Tan AR, et al. Phase II randomized clinical trial evaluating neoadjuvant chemotherapy regimens with weekly paclitaxel or eribulin followed by doxorubicin and cyclophosphamide in women with locally advanced HER2-negative breast cancer: NSABP Foundation Study FB-9. Breast Cancer Res Treat. 2015;152(2):399-405. doi:10.1007/s10549-015-3466-4

38. Yardley DA, Shipley D, Zubkus J, et al. A randomized phase ii study of eribulin/cyclophosphamide or docetaxel/cyclophosphamide as neoadjuvant therapy in operable HER2-negative breast cancer. Clin Breast Cancer. 2019;19(1):1-9. doi:10.1016/j.clbc.2018.08.006

39. McIntyre K, O’Shaughnessy J, Schwartzberg L, et al. Phase 2 study of eribulin mesylate as first-line therapy for locally recurrent or metastatic human epidermal growth factor receptor 2-negative breast cancer. Breast Cancer Res Treat. 2014;146(2):321-328. doi:10.1007/ s10549-014-2923-9

40. Ortega V, Anton A, Garau I, et al. Phase II, multicenter, single-arm trial of eribulin as first-line therapy for patients with aggressive taxane-pretreated HER2-negative metastatic breast cancer: the MERIBEL study. Clin Breast Cancer. 2019;19(2):105-112. doi:10.1016/j.clbc.2018.12.012

41. Takashima T, Tokunaga S, Tei S, et al. A phase II, multicenter, single-arm trial of eribulin as first-line chemotherapy for HER2-negative locally advanced or metastatic breast cancer. SpringerPlus. 2016;5(1):164. doi:10.1186/s40064-016-1833-1

42. Kimura K, Iwamoto M, Tanaka S, et al. A phase II, multicenter, single-arm trial of eribulin as first- or second-line chemotherapy for HER2-negative advanced or metastatic breast cancer: evaluation of efficacy, safety, and patient-reported outcomes. Cancer Chemother Pharmacol. 2018;81(5):923-933. doi:10.1007/s00280-018-3567-y

43. Park YH, Im S-A, Kim S-B, et al. Phase II, multicentre, randomised trial of eribulin plus gemcitabine versus paclitaxel plus gemcitabine as first-line chemotherapy in patients with HER2-negative metastatic breast cancer. Eur j Cancer. 2017;86:385-393. doi:10.1016/j.ejca.2017.10.002

44. Tolaney SM, Barroso-Sousa R, Keenan T, et al. Effect of eribulin with or without pembrolizumab on progression-free survival for patients with hormone receptor-positive, ERBB2 -negative metastatic breast cancer. JAMA Oncology. 2020;6(10):1598. doi:10.1001/ jamaoncol.2020.3524

45. Inoue K, Takahashi M, Mukai H, et al. Effectiveness and safety of eribulin in Japanese patients with HER2-negative, advanced breast cancer: a 2-year post-marketing observational study in a real-world setting. Invest New Drugs. 2020;38(5):1540-1549. doi:10.1007/s10637-019-00890-5

46. Pernas S, Martin M, Kaufman PA, et al. Balixafortide plus eribulin in HER2-negative metastatic breast cancer: a phase 1, single-arm, dose-escalation trial. Lancet Oncol. 2018;19(6):812-824. doi:10.1016/S1470-2045(18)30147-5

47. Lee JS, Yost SE, Blanchard S, et al. Phase I clinical trial of the combination of eribulin and everolimus in patients with metastatic triple-negative breast cancer. Breast Cancer Res. 2019;21(1):119. doi:10.1186/s13058-019-1202-4 
48. Yonemori K, Shimomura A, Yasojima H, et al. A phase I/II trial of olaparib tablet in combination with eribulin in Japanese patients with advanced or metastatic triple-negative breast cancer previously treated with anthracyclines and taxanes. Eur j Cancer. 2019;109:84-91. doi:10.1016/j.ejca.2018.11.014

49. Kashiwagi S, Asano Y, Goto W, et al. Use of Tumor-infiltrating lymphocytes (TILs) to predict the treatment response to eribulin chemotherapy in breast cancer. PLoS One. 2017;12(2):e0170634. doi:10.1371/journal.pone.0170634

50. Miyagawa Y, Araki K, Bun A, et al. Significant association between low baseline neutrophil-to-lymphocyte ratio and improved progression-free survival of patients with locally advanced or metastatic breast cancer treated with eribulin but not with nab-paclitaxel. Clin Breast Cancer. 2018;18(5):400-409. doi:10.1016/j. clbc.2018.03.002

51. Miyoshi Y, Yoshimura Y, Saito K, et al. High absolute lymphocyte counts are associated with longer overall survival in patients with metastatic breast cancer treated with eribulin - but not with treatment of physician's choice-in the EMBRACE study. Breast Cancer. 2020;27(4):706-715. doi:10.1007/s12282-020-01067-2

52. Chinese Anti-Cancer Association Breast Cancer Committee. Chinese Anti-Cancer Association breast cancer diagnosis and treatment guidelines [Chinese Language]. China Oncol. 2019;29(8).

53. Yanmei L. Guidelines of Chinese Society of Clinical Oncology (CSCO): Breast Cancer. China; 2020.

54. Wozniak KM, Wu Y, Farah MH, Littlefield BA, Nomoto K, Slusher BS. Neuropathy-inducing effects of eribulin mesylate versus paclitaxel in mice with preexisting neuropathy. Neurotox Res. 2013;24(3):338-344. doi:10.1007/s12640-013-9394-3

55. Wozniak KM, Nomoto K, Lapidus RG, et al. Comparison of neuropathy-inducing effects of eribulin mesylate, paclitaxel, and ixabepilone in mice. Cancer Res. 2011;71(11):3952-3962. doi:10.1158/ 0008-5472.CAN-10-4184
56. Benbow SJ, Wozniak KM, Kulesh B, et al. Microtubule-targeting agents eribulin and paclitaxel differentially affect neuronal cell bodies in chemotherapy-induced peripheral neuropathy. Neurotox Res. 2017;32(1):151-162. doi:10.1007/s12640-017-9729-6

57. Vahdat LT, Garcia AA, Vogel C, et al. Eribulin mesylate versus ixabepilone in patients with metastatic breast cancer: a randomized Phase II study comparing the incidence of peripheral neuropathy. Breast Cancer Res Treat. 2013;140(2):341-351. doi:10.1007/ s10549-013-2574-2

58. Zuo T, Zeng H, Li H, et al. The influence of stage at diagnosis and molecular subtype on breast cancer patient survival: a hospital-based multi-center study. Chin J Cancer. 2017;36(1):84. doi:10.1186/ s40880-017-0250-3

59. NCCN Clinical Practice Guidelines in Oncology. Breast Cancer. NCCN Evidence Blocks. 2020; Available from: https://www.nccn. org/professionals/physician_gls/pdf/breast_blocks.pdf. Accessed October 1, 2020..

60. National Comprehensive Cancer Network Evidence Blocks User Guide; 2020. Available from: https://www.nccn.org/evidenceblocks/ pdf/EvidenceBlocksUserGuide.pdf. Accessed October 1, 2020.

61. Smith J, Irwin A, Jensen L, et al. Phase II study of eribulin mesylate administered biweekly in patients with human epidermal growth factor receptor-2-negative metastatic breast cancer. Clin Breast Cancer. 2020;20(2):160-167. doi:10.1016/j.clbc.2019.09.007

62. Wikipedia. Avalablefrom: https://en.wikipedia.org/wiki/Eribulin.

\section{Publish your work in this journal}

Breast Cancer - Targets and Therapy is an international, peer-reviewed open access journal focusing on breast cancer research, identification of therapeutic targets and the optimal use of preventative and integrated treatment interventions to achieve improved outcomes, enhanced survival and quality of life for the cancer patient.
The manuscript management system is completely online and includes a very quick and fair peer-review system, which is all easy to use. Visit http://www.dovepress.com/testimonials.php to read real quotes from published authors. 